\begin{tabular}{|c|l|}
\hline Title & Design of quasi-millimeter wave leaf-shaped bowtie array antenna for UWB applications \\
\hline Author(s) & Y amamoto, Manabu; Tokuyama, Daisuke; Nojima, Toshio \\
\hline Citation & $\begin{array}{l}2010 \text { IEEE Antennas and Propagation Society International Symposium (APSURSI), 1-4 } \\
\text { https://doi.org/10.1109/APS.2010.5561106 }\end{array}$ \\
\hline Issue Date & 2010-07-11 \\
\hline Doc URL & http://hdl.handle.net/2115/47284 \\
\hline Type & proceedings(author version) \\
\hline File Information & APSURSI 2010_1-4.pdf \\
\hline
\end{tabular}

Instructions for use 


\title{
Design of Quasi-Millimeter Wave Leaf-Shaped Bowtie Array Antenna for UWB Applications
}

\author{
Manabu Yamamoto, Daisuke Tokuyama and Toshio Nojima
}

Graduate School of Information Science and Technology, Hokkaido University

Kita 14, Nishi 9, Kita-ku, Sapporo, 060-0814 Japan

\section{Introduction}

Ultra-wideband (UWB) radio technology has recently attracted considerable attention for various applications, such as short-range high-speed communication, sensor networks, radar and location tracking. In 2002, the Federal Communications Commission (FCC) announced its decision to allow the unlicensed use of the frequency band from 3.1 to $10.6 \mathrm{GHz}$ for UWB communication systems [1]. The FCC also allocated the quasi-millimeter wave band of 22 to $29 \mathrm{GHz}$ for radar applications. As a practical use of UWB radar systems, short-range (SR) automotive radar operating in the quasi-millimeter wave band has been receiving increased interest in recent years.

Since the allocation of the UWB bands by the FCC, many research groups have proposed various types of UWB antennas. In particular, planar-type UWB antennas have attracted significant research power in the past few years [2]-[4]. Most of planar-type UWB antennas reported up to now have omnidirectional radiation patterns and low directivity, approximately $0-3 \mathrm{dBi}$. On the other hand, unidirectional and high gain antennas with ultra-wideband characteristics are required in order to realize the quasi-millimeter wave UWB radar systems including the SR automotive radar.

To meet these requirements, this paper presents an UWB array antenna having unidirectional radiation characteristics and high gain over the quasi-millimeter wave band of 22 to $29 \mathrm{GHz}$. The developed array antenna is composed of leaf-shaped bowtie antennas, which are previously proposed by the authors [4]. In order to demonstrate effective performance of the proposed configuration, fundamental characteristics of the antenna array obtained by FDTD analysis and measurements are presented.

\section{Antenna Design}

Fig. 1 illustrates the configuration and coordinate system of the proposed array antenna. Two pairs of radiating elements are printed on top and bottom sides of a dielectric substrate having the thickness of $h$, relative permittivity of $\varepsilon_{r}$ and dielectric loss of $\tan \delta$. The side lengths of the substrate are designated as $L_{s}$ and $W_{s}$. The radiating elements are placed with the separation of $S$. In the proposed configuration, leaf-shaped bowtie antenna is adopted as the radiating elements. As shown in Fig. 2, the leaf-shaped antenna [4] is designed by rounding the corner of the square copper sheet with the curvature radius of $R_{s}$ and the central angle of $\alpha$. The side length of the square shape is denoted by $L_{e}$. The radiating elements are excited by a feeding circuit consisting of a tapered microstrip line and a parallel strip line T-junction. In order to 
obtain unidirectional radiation characteristics, a back reflector, having the side lengths of $L_{r}$ and $W_{r}$, is placed underneath the antenna substrate. The separation between the substrate and the reflector is denoted by $d$. In the following analysis and measurements, the structural parameters of the proposed antenna are set to values shown in Table 1. These parameters are designed so that the operating frequency of the array antenna is the quasi-millimeter wave band from 22 to $29 \mathrm{GHz}$.

\section{Simulation and Experimental Results}

In the first place, a single-element leaf-shaped bowtie antenna is analyzed by using FDTD method in order to confirm the fundamental characteristics of the antenna. In the analysis, it is assumed that the antenna is excited by a delta-gap voltage source connected to the center of the radiating element as shown in Fig. 2.

Frequency response of the input impedance and the actual gain observed in the $+z$-direction is shown in Fig. 3 and Fig. 4, respectively. It is seen that the real part of the input impedance is around $180 \Omega$, which is close to that of the self-complementary antenna. The actual gain is almost constant value of around $2.5 \mathrm{dBi}$, which is slightly higher than that of the conventional half-wavelength dipole antenna.

Next, the performances of the 2-element leaf-shaped bowtie array antenna at the quasi-millimeter wave band are evaluated by FDTD analysis and measurement. The prototype antenna was fabricated by using ARLON DiClad 880 dielectric substrate.

Fig. 5 shows the simulated and measured reflection coefficient versus frequency. In the measurement, the prototype antenna was connected to the vector network analyzer (Agilent HP8510C) through a $50 \Omega$ semirigid coaxial cable and K-connectors. The time-domain gating technique on the network analyzer is utilized for the purpose of removing unwanted reflections caused by the connectors. The measured reflection is less than $-12 \mathrm{~dB}$ over the frequency range from 22 to $29 \mathrm{GHz}$. On the other hand, the simulated reflection is larger than $-10 \mathrm{~dB}$ excepting the frequency around $27 \mathrm{GHz}$. The discrepancy between the measured and simulated results may be attributed to the effect of the conductor loss of the radiating element and the coaxial cable.

The frequency response of actual gain simulated and measured in the $+z$-direction is shown in Fig. 6. As for the measured result, the actual gain of 8 to $10 \mathrm{dBi}$ is obtained. It is seen that the gain is improved by around $6 \mathrm{~dB}$ in comparison with the single-element case shown in Fig. 4. The discrepancy between the simulation and measurement becomes larger with the increase of the frequency. This may be due to the effect of the conductor loss as described in the case of the reflection coefficient.

The co-polarization patterns in the H-plane ( $x z$-plane) and E-plane ( $y z$-plane) are shown in Fig. 7 and Fig. 8, respectively. In both figures, the results are normalized by the maximum gain, and are observed at 22, 25 and $29 \mathrm{GHz}$. As can be seen from the figures, simulated and measured results are in good agreement. It is also confirmed that unidirectional radiation characteristics are obtained over the frequency band of 22 to 29GHz. The shapes of the E-plane patterns are not symmetrical with respect to the broadside direction. This may be attributed to the presence of the feeding circuit. 


\section{Conclusions}

A quasi-millimeter wave UWB array antenna has been presented in this paper. As a basic component of the proposed array antenna, a leaf-shaped bowtie element is adopted, and its fundamental characteristics are revealed with the FDTD results. 2-element quasi-millimeter wave UWB array antenna is composed of the leaf-shaped bowtie element, and its performances at the quasi-millimeter wave band of 22 to $29 \mathrm{GHz}$ is evaluated by the FDTD analysis measurements. Measured and simulated results validate the effective performance of the proposed configuration.

\section{References}

[1] FCC, "First Report and Order, Revision of Part 15 of the Commission's Rules Regarding Ultra-Wideband Transmission System", FCC02-48, Apr. 2002.

[2] X. H. Wu and Z. N. Chen, "Comparison of Planar Dipoles in UWB Applications", IEEE Trans. Antennas Propag., Vol. 53, No. 6, pp.1973-1983, June 2005.

[3] Y. D. Dong, et al., "Analysis of Planar Ultrawideband Antennas with On-Ground Slot Band-Notched Structures", IEEE Trans. Antennas Propag., Vol. 57, No. 7, pp.1886-1893, July 2009.

[4] M. Ameya, M. Yamamoto and T. Nojima, "An Omnidirectional UWB Printed Dipole Antenna with Small Waveform Distortion", Proc. of Progress In Electromagnetics Research Symposium 2006, 4P3, p.515, Aug. 2006.

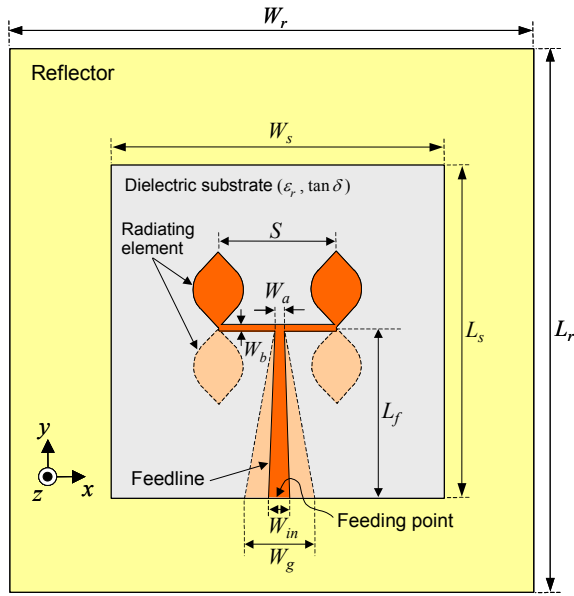

(a) Top view

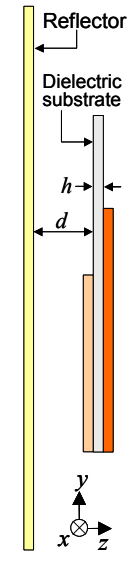

(b) Side view

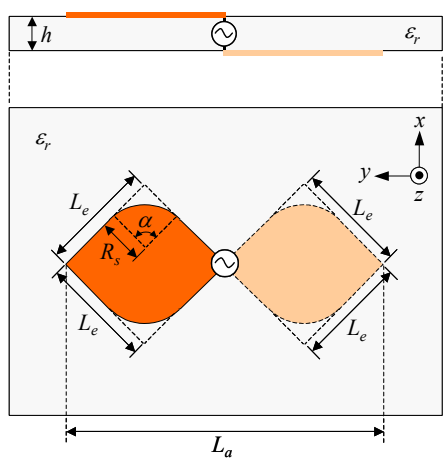

Fig. 2 Leaf-shaped bowtie antenna.

Fig. 1 Antenna configuration.

Table 1 Structural parameters.

\begin{tabular}{|c|c|c|c|c|c|c|c|}
\hline$L_{s}[\mathrm{~mm}]$ & $W_{s}[\mathrm{~mm}]$ & $h[\mathrm{~mm}]$ & $\varepsilon_{r}$ & $\tan \delta$ & $L_{r}[\mathrm{~mm}]$ & $W_{r}[\mathrm{~mm}]$ & $d[\mathrm{~mm}]$ \\
\hline 20 & 20 & 0.38 & 2.17 & 0.0085 & 40 & 40 & 2.0 \\
\hline
\end{tabular}

\begin{tabular}{|c|c|c|c|c|c|c|c|c|c|}
\hline$R_{s}[\mathrm{~mm}]$ & $\alpha[\mathrm{deg}]$ & $L_{e}[\mathrm{~mm}]$ & $L_{a}[\mathrm{~mm}]$ & $S[\mathrm{~mm}]$ & $W_{i n}[\mathrm{~mm}]$ & $W_{g}[\mathrm{~mm}]$ & $W_{a}[\mathrm{~mm}]$ & $W_{b}[\mathrm{~mm}]$ & $L_{f}[\mathrm{~mm}]$ \\
\hline 1.5 & 90 & 2.8 & 8.0 & 6.0 & 1.1 & 6.0 & 1.0 & 0.3 & 10 \\
\hline
\end{tabular}




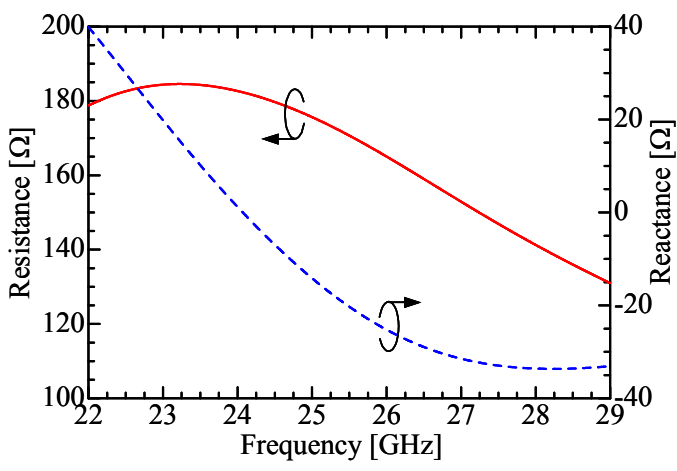

Fig. 3 Input impedance of single-element leaf-shaped bowtie antenna.

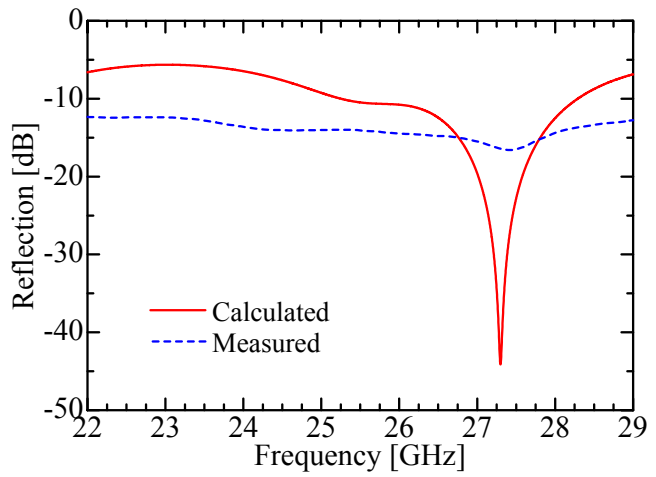

Fig. 5 Reflection coefficient of 2-element leaf-shaped bowtie array antenna.

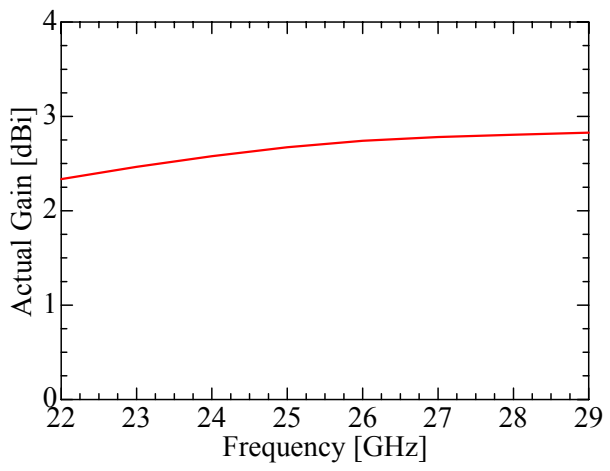

Fig.4 Actual gain of single-element leaf-shaped bowtie antenna.

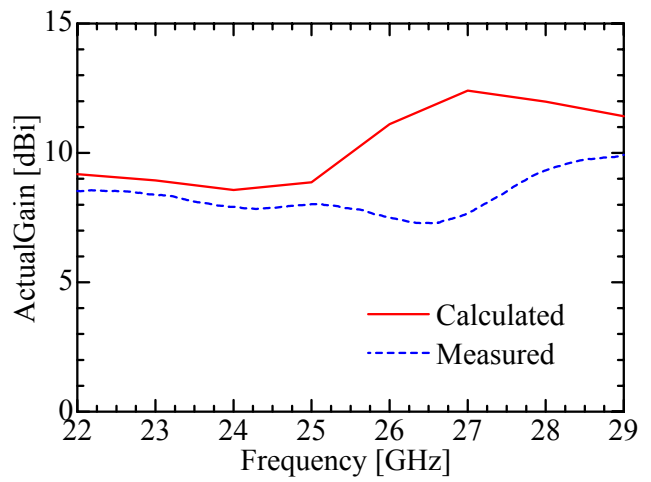

Fig. 6 Actual gain of 2-element leafshaped bowtie array antenna.

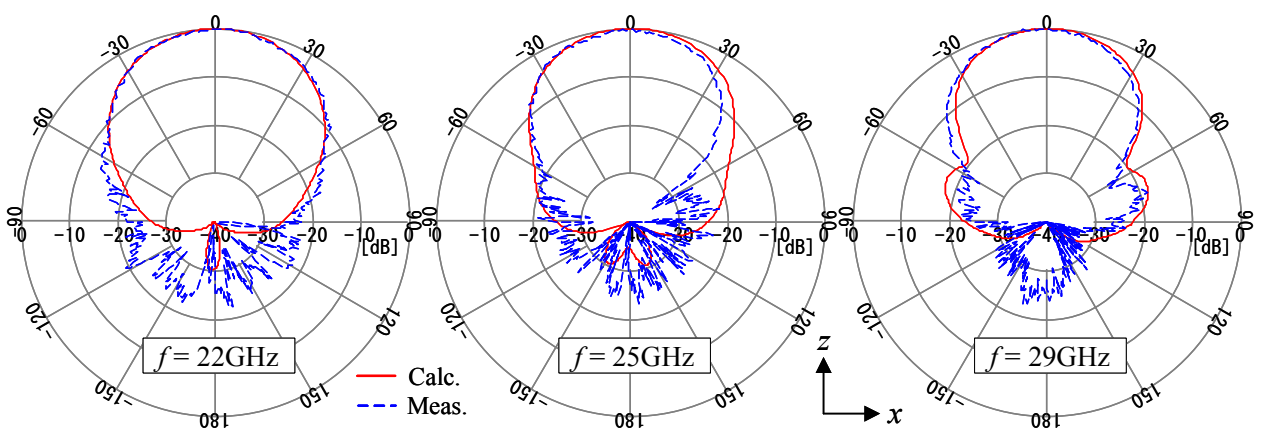

Fig. 7 Radiation patterns in $x z$-plane (co-polarization).

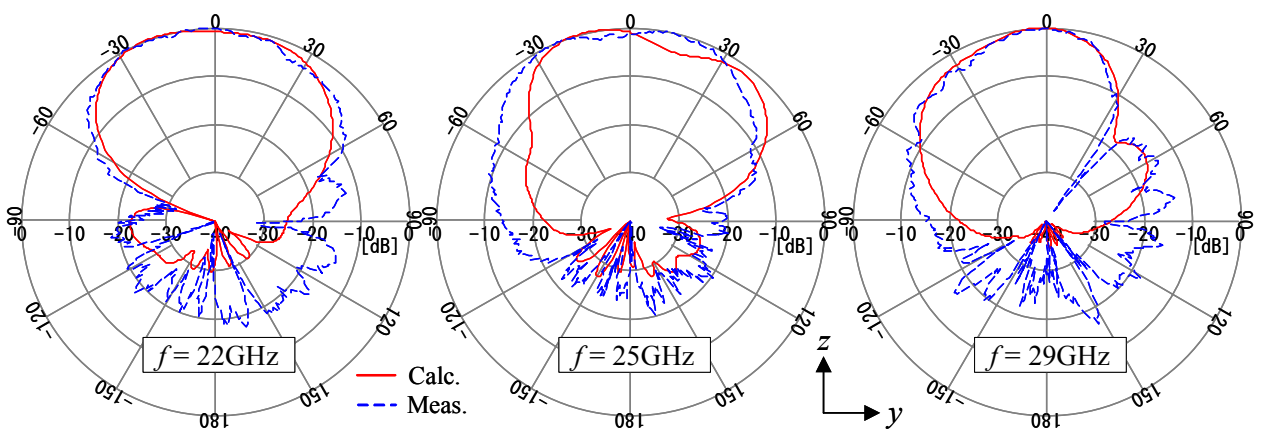

Fig. 8 Radiation patterns in $y z$-plane (co-polarization). 